\title{
Proliferation studies on chromosome preparations of bone marrow in hematological disease
}

\author{
J-U. WALTHER, I. POHL, A. RAUSCH and M. FUEHRER \\ Kinderklinik und Kinderpoliklinik im Dr. v. Haunerschen Kinderspital, der Ludwig-Maximilians-Universitaet \\ Muenchen, Lindwurmstr. 4, D-80336 München, Germany
}

Received March 2, 2006; Accepted May 24, 2006

\begin{abstract}
The growth rate of neoplastic cells has been the subject of numerous scientific and diagnostic approaches. The study presented here analyses the relationship between mitotic activity in standardised cytogenetic bone marrow preparations from three haematological diseases and diagnostic and clinical parameters, most importantly the outcome. The disorders studied were: Acute lymphoblastic leukemia (ALL) $(\mathrm{N}=107)$, chronic myeloid leukemia $(\mathrm{CML})(\mathrm{N}=166)$ and aplastic anemia in childhood (AA) $(\mathrm{N}=39)$. A strict protocol of quantitative standardisation of cytogenetic slides was adhered to ensuring comparability both cross-sectionally and longitudinally. The samples were studied after short-term incubation without mitogenic in vitro stimuli. The most important findings include: i) ALL: Immunological subtypes can be differentiated according to their proliferation profile; there is a striking difference between childhood and adult ALL in proliferation activity; most importantly initial proliferation is much higher in patients who will relapse than in those with stable remission. ii) CML: Philadelphia-positive CML shows proliferation activities quite distinct from Philadelphia-negative CML; however there is only a small change in the proliferative activity from the chronic phase to the accelerated phase or blast crisis. iii) AA: Very low proliferation scores rise quickly to near normal levels during immunosuppressive therapy in most patients. Higher levels at diagnosis are associated with a faster and better response to therapy. In conclusion, assessment of the proliferative activity in cytogenetic preparations made from bone marrow samples of patients with haematological disease may add valuable information as to diagnostic sub-groups and clinical course and may contribute to therapeutic decisions.
\end{abstract}

Correspondence to: Dr J.-U. Walther, Kinderklinik und Kinderpoliklinik im Dr. v. Haunerschen Kinderspital, der LudwigMaximilians-Universitaet Muenchen, Lindwurmstr. 4, D-80336 München, Germany

E-mail: ju.walther@med.uni-muenchen.de

Key words: proliferation, mitoses, bone marrow, malignancy, leukemia, aplastic anemia, prognosis

\section{Introduction}

Neoplastic disease may be described as disturbed homeostasis of tissue-cell turnover favouring growth over apoptosis. Powerful methods exist to identify and measure this imbalance in individual tissue samples particularly in haematology (1-4) in order to describe the neoplastic dynamics in individual patients and to correlate findings with clinical parameters such as disease type, cause and outcome. Most of these methods, however, are technically involved and/or require sophisticated equipment. We studied the mitotic activity observed on conventional cytogenetic slides prepared in routine work-up of leukemia and aplastic anemia (AA) for the potential use of this by-product of an established diagnostic tool to obtain additional diagnostic and prognostic information.

\section{Patients and methods}

Probands. Acute lymphoblastic leukaemia (ALL): One hundred and seven consecutive cases (97 children, ten adults: $>15$ years), (93 c-ALL; 11 t-ALL; 3 undifferentiated ALL).

Chronic myeloid Leukaemia (CML): 166 consecutive cases (Philadelphia-positive $\mathrm{N}=155$ : 136 in chronic phase; 19 in $\mathrm{AP} / \mathrm{BC}$, Philadelphia negative $\mathrm{N}=11)$.

Acquired aplastic anaemia (AA): 39 consecutive cases, children studied at diagnosis $(\mathrm{N}=39)$, day 112 after starting immunosuppressive treatment (IST: anti-thymocyte globulin, Cyclosporine A) $(\mathrm{N}=28)$, six months $(\mathrm{N}=17), 12$ months $(\mathrm{N}=5)$.

Controls: Twenty-four bone marrow samples from 15 healthy donors (for transplantation). Informed consent was obtained from all probands.

Cultivation, slide preparation. Chromosomal preparations from bone marrow (BM) aspirates were made according to routine methods (5). Greatest care was given to the quantitative standardisation, i.e. a constant amount of cells $\left(10^{7}\right)$ was inoculated into the culture medium ( $8 \mathrm{ml} \mathrm{RPMI}$ $1640+2 \mathrm{ml}$ fetal calf serum) and a constant volume of cell suspension $(0.15 \mathrm{ml})$ spread on the final slide after a rigorously standardised protocol of hypotonic treatment and washings. If the result was unsatisfactory for karyotyping, slides with higher or lower metaphase counts, prepared in parallel, were evaluated, but only the 'standard slide' was used for proliferation analysis. 
Table I. Definition of the semi-quantitative proliferation score as a reflection of the metaphase count.

\begin{tabular}{lc}
\hline Score & Metaphases per slide \\
\hline 0 & 0 \\
1 & $1-4$ \\
2 & $5-20$ \\
3 & $>20$ \\
\hline
\end{tabular}

\section{Proliferation of unstimulated bm aspirates in healthy donors}

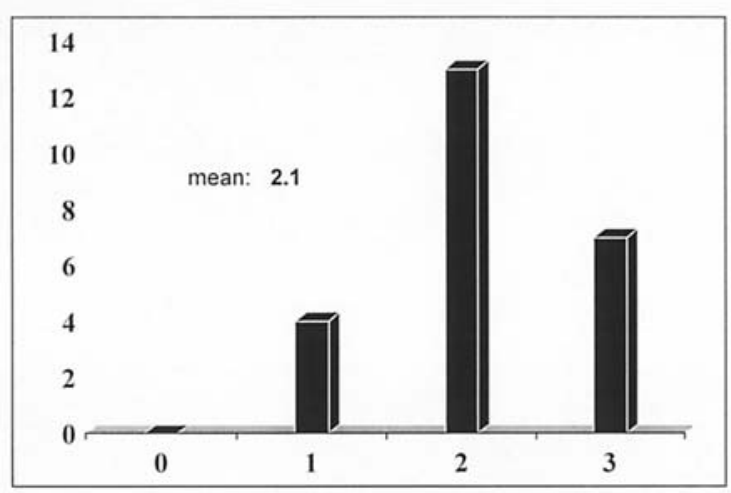

Figure 1. Proliferation profile in $24 \mathrm{bm}$ samples of 15 healthy donors.

\section{Proliferation Profile of ALL}

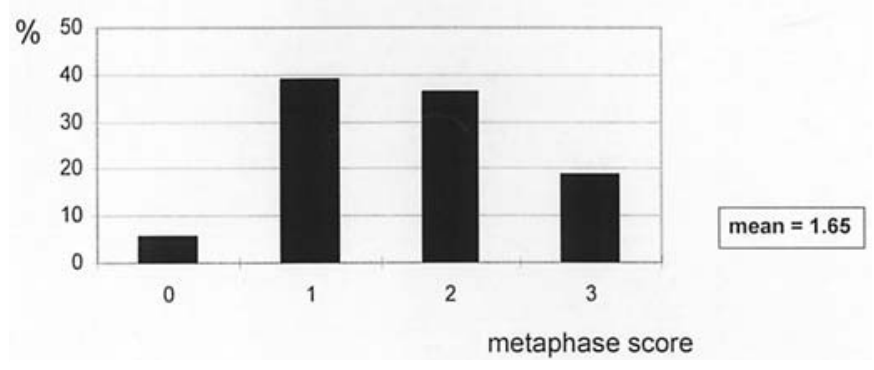

Figure 2. Proliferation profile in ALL in each of the cases.

Proliferation measurement. Metaphases were counted per slide and documented as a semi-quantitative score (Table I). Profiles resulted from these scores depicting the distribution of proliferation at diagnosis and specified intervals of monitoring.

For a better overview and comparison average values were taken from each profile and documented as mean proliferation scores.

In leukemia patients only the samples obtained at diagnosis were evaluated. In AA, however, we additionally performed follow-up studies at day 112, and after 6 and 12 months of treatment, respectively (Fig. 1) according to the treatment protocol.
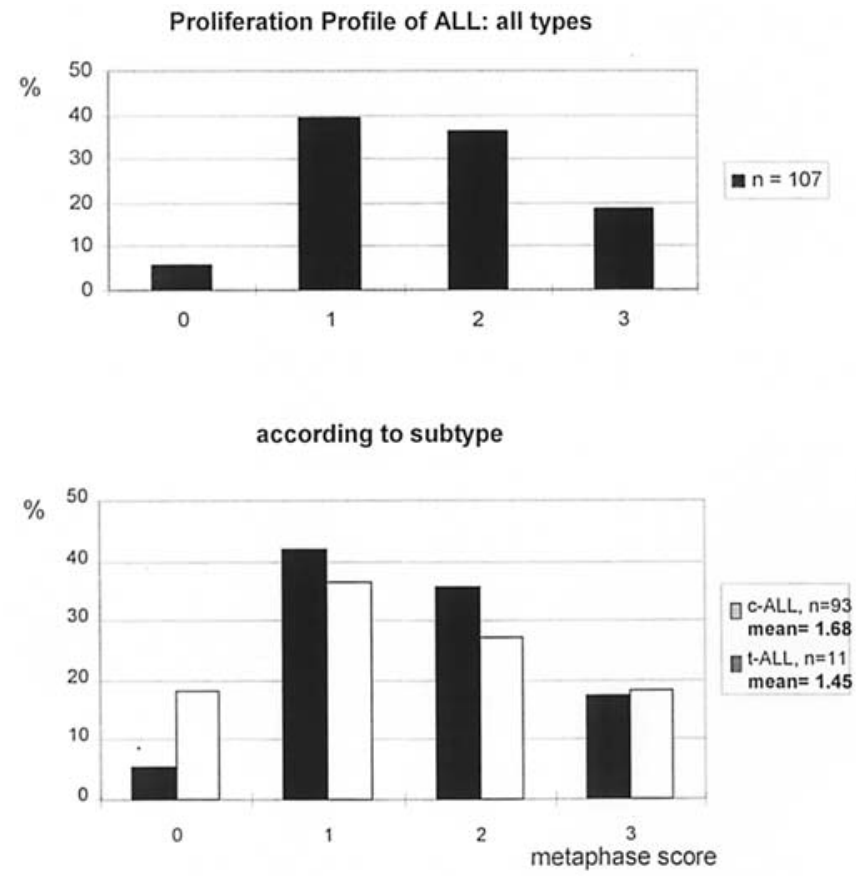

Figure 3. Proliferation profile in ALL according to subtypes.

\section{Results}

Controls. The bone marrow samples of 24 healthy donors showed a near 'normal' distribution of proliferation scores (Fig. 2) with a mean value of 2.1. There was no sample with nil proliferation, i.e. on all slides metaphases were identified.

Acute lymphoblastic leukemia (ALL). Fig. 3 shows the distribution of proliferation scores in all ALL patients investigated. As compared to the controls the profile is clearly shifted towards the left with $5 \%$ of the samples showing no metaphases. The mean proliferation score is 1.65. Sub-groups include: i) Immunological subtype: An even greater reduction of proliferation is found for T-ALL $(\mathrm{N}=11)$ with an $18 \%$ failure rate and an average score of 1.45 (note small numbers), while c-ALL as the predominant subgroup reflects the overall result (Fig. 4). ii) Age: A much more active proliferation is found for the adult subgroup (again a small number of ten). The majority of adult patients reach high proliferation rates with an average of 2.4 vs 1.6 for the children (Fig. 5). iii) Proliferation and outcome: The most striking difference was found between patients with relapse and those in continuous remission: the former showed clearly higher proliferation at diagnosis (mean = 2.4) than the majority of ALL-patients without relapse $($ mean $=1.5)($ Fig. 6).

Chronic myeloid leukemia (CML). The proliferation profile of CML closely resembles that of normal controls with a slight shift towards the left and a corresponding decrease of the mean value to 1.9. In four percent no metaphases were identified on the standardised slides (Fig. 7). Influence of subtype includes: i) Philadelphia-negative CML: Eleven patients showed a clearly lower degree of proliferation 


\section{Proliferation profile in children with ALL accordig to outcome}

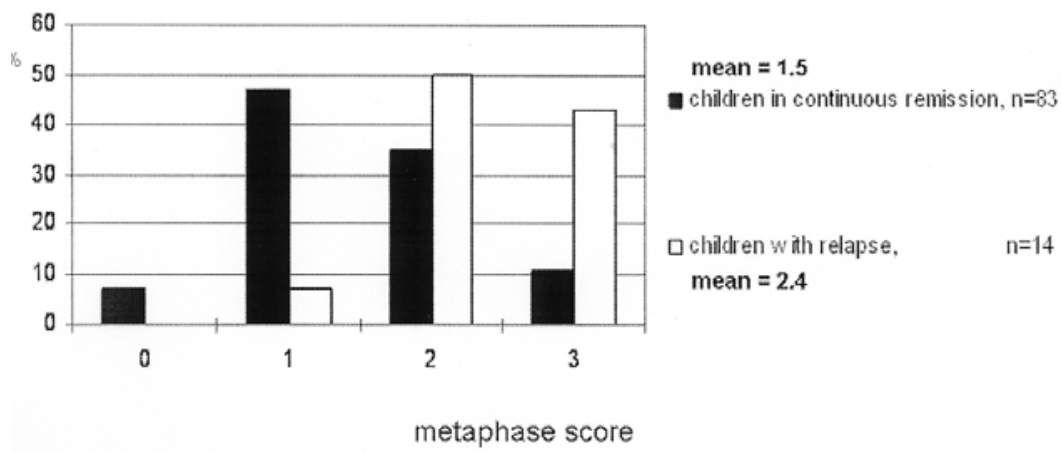

Figure 4. Proliferation profile in ALL (at diagnosis) according to outcome.

\section{CML proliferation profile}

all phases $/ \mathrm{N}=155$

$\%$

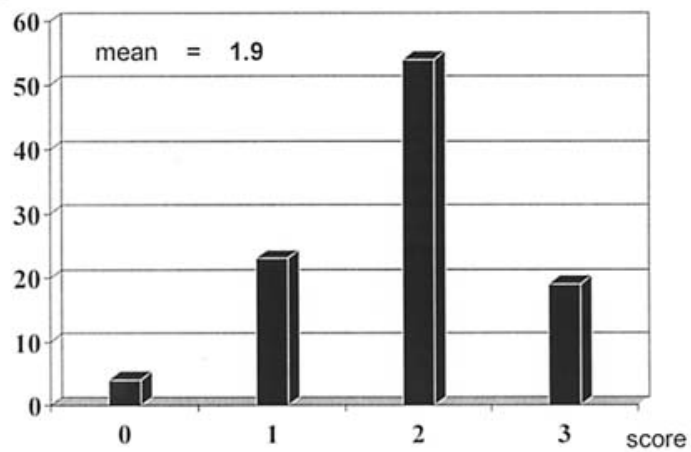

Figure 5. Proliferation profile in CML in each of the phases.

CML proliferation profile

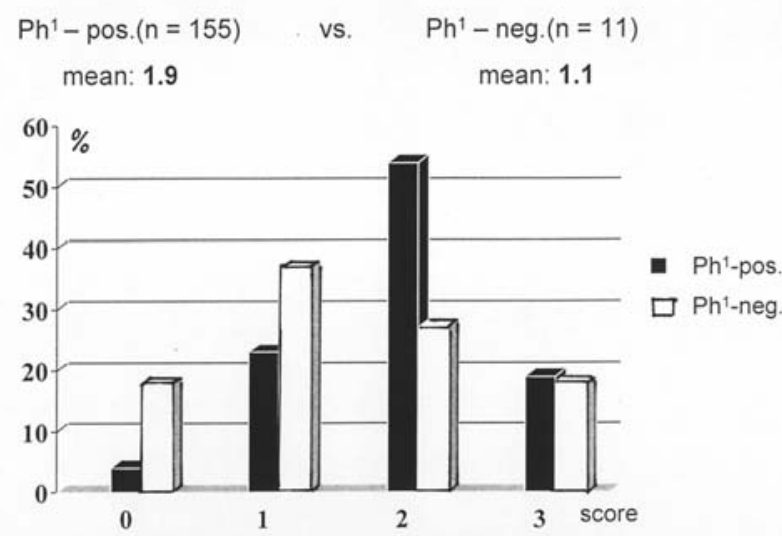

Figure 6. Proliferation profile in CML according to the $\mathrm{Ph}^{1}$ status.

(mean of 1.1) and $18 \%$ of proliferation failures (Fig. 7). ii) Stage: Bone marrow samples from the acute phase or blast crisis showing a higher number of metaphases with a mean score of 2.5 vs 1.8 for the chronic phase (Fig. 8).
CML proliferation profile

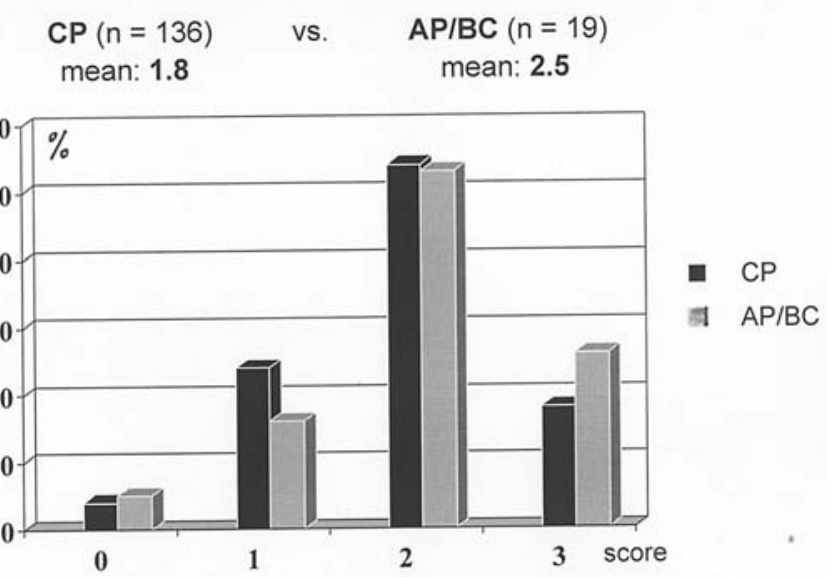

Figure 7. Proliferation profile in CML according to the phase of the disease.

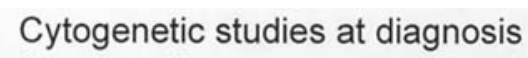
and follow-up of immunosuppressive therapy

n

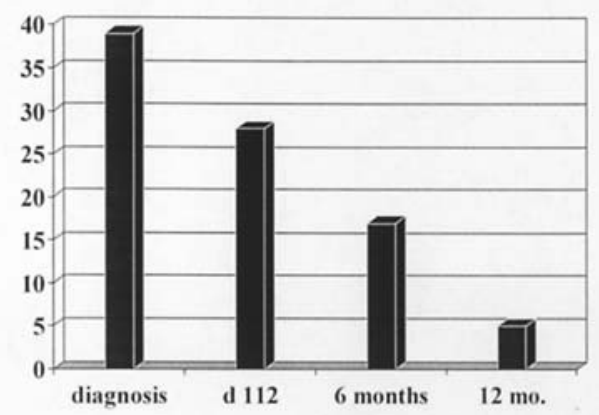

Figure 8. Sequential proliferation studies in the AA cohort

Acquired aplastic anemia (AA). At diagnosis the 39 pediatric patients presented a unique proliferation profile with a pronounced shift to the left and 14 of the 39 (36\%) showed no proliferation at all. The mean proliferation score is 1.1 (Fig. 9). 
$\mathrm{AA}$ at Diagnosis

Mean Score: 1.1

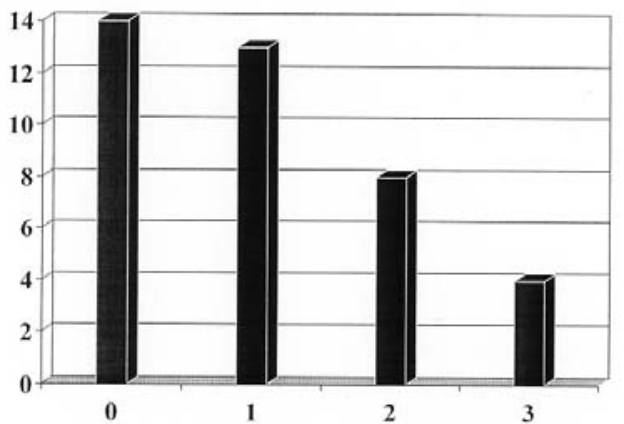

Figure 9. Proliferation profile in AA at diagnosis.

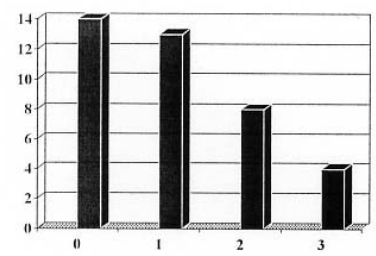

diagnosis

mean score:

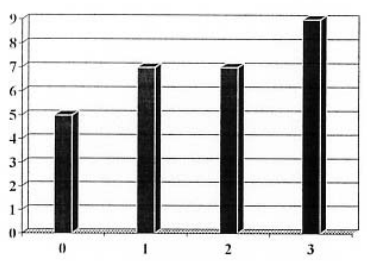

day 112

mean score: 1.7

6 months

mean score: 1.9

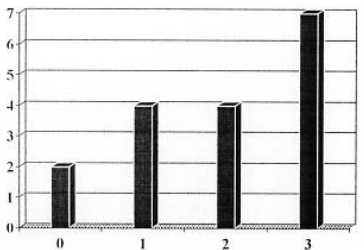

Figure 10. Proliferation profiles in AA during follow-up.

A striking change occurred after three months of treatment with immunosuppression and cytokines: only five among the 28 patients (18\%) still failed to show any proliferation, the mean score reaching 1.7 (Fig. 9).

After three more months lack of metaphases was observed in only two among $17(12 \%)$ patients with a mean score of 1.9 (Fig. 10).

For better comparison Fig. 11 shows a breakdown of the mean proliferation scores at diagnosis in patients with early, late and no treatment response, the latter again differentiated according to the stage of follow-up at which no response was noted.

\section{Mean initial proliferation score according to speed of response}

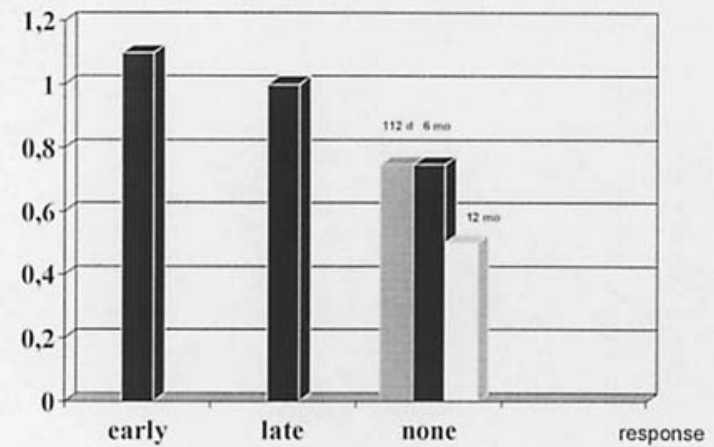

Figure 11, Mean initial proliferations scores in AA according to the speed of response.

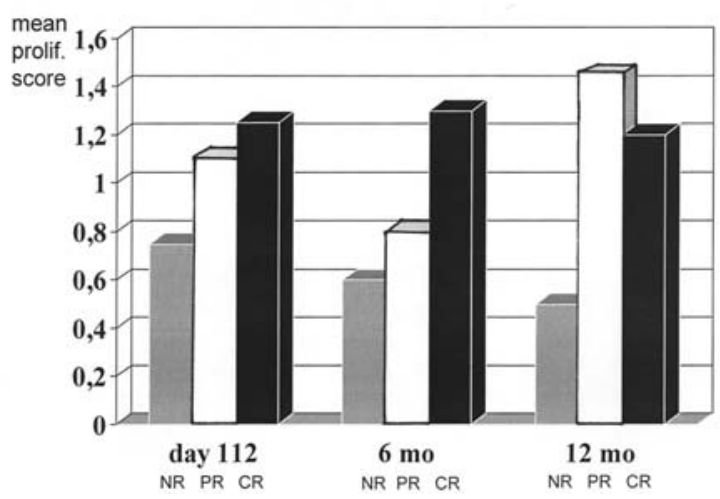

Figure 12. Mean initial proliferation scores in AA according to response status at milestones.

A clear trend is seen towards higher proliferation in cases with a stronger and earlier response.

This correlation is visualised again in greater detail in Fig. 12. Here the mean proliferation scores at diagnosis are plotted against the response status (no response, partial and complete response) at the three time intervals. Again low proliferation is clearly associated with poor treatment response and vice versa. At 12 months this correlation is distorted probably due to the low number of probands $(\mathrm{N}=5)$.

\section{Discussion}

Chromosomal analysis of acute and chronic leukemia has become an established component of diagnosis and followup. Its main clinical significance is for sub-classification, identification of special risk factors and thus the planning of risk-adapted therapy as well as longitudinal follow-up $(5,6)$. Despite the emergence of molecular techniques with much higher sensitivity (FISH and PCR) the advantage of classical 
cytogenetic methodology still lies in the global genetic characterisation of the neoplastic clone only to be challenged by the advent of expression profiling techniques (7).

Besides the qualitative information provided by the karyotype, a cytogenetic preparation represents a 'snapshot' of proliferation of the tissue studied. Obviously the in vivo situation may be modified - if not falsified - by in vitro conditions such as long cultivation time, selection of tissue components or even clones etc. Both sources of error have been minimised in the study presented here as preparation was based on short-term (24-h) culture and the absence of any stimulating agents. Consequently the metaphases identified and counted cannot be classified as to their tissue origin or clonal identity. In acute leukemia at diagnosis the large majority of bone marrow cells belong to the neoplastic population, thus it is reasonable to assume that mitoses will predominantly belong to this cell-type. The situation may be quite different during follow-up when chemotherapy may dramatically change the proportions of neoplastic and normal hemopoiesis.

Nevertheless this investigation primarily aims to identify the proliferation characteristics of various haematological diseases at diagnosis regardless of the origin of dividing cells counted.

Neoplasia does not necessarily entail a higher mitotic 'speed'. Cells accumulate due to the shift from differentiation to proliferation. In ALL for instance the turnover time of a dividing malignant cell may show low levels in certain subtypes (8). This may be one of the reasons why particularly in this disease the mitotic yield of uncultivated or short-term culture preparations may be notoriously low as opposed to other types of bone marrow malignancy.

This variability and the experience from routine laboratory studies led us to systematically study the information on proliferation kinetics contained in chromosomal preparations.

Normal controls. In clinical cytogenetics some experience has been gained with the use of bone marrow as the source of constitutional karyotypes in case of urgent chromosomal diagnosis such as severely ill newborns suspected of aneuploidy. The use of bone marrow allows direct or shortterm culture preparation of chromosomes without the time consuming stimulation by mitogens. This experience, however, is largely restricted to neonatology with its well-known higher degree of cell turnover. Therefore we had to establish a normal range of proliferation scores in a group of bone marrow donors of ages between five and thirty-five years. The distribution of proliferation found was in the range that qualified as moderate to good in our routine laboratory practice. Notably, there were no mitotic failures (Fig. 2).

ALL. ALL being the most frequent single neoplastic disease in childhood has been renowned for its poor cytogenetic results both in numbers and structural properties of metaphases. Indeed experienced cytogeneticists are able to 'suspect' ALL from its chromosomal appearance even before any typical chromosomal abnormality has been identified. There are numerous technical refinements to circumvent this notorious difficulty of scanty and ill defined metaphases in ALL (9). Nevertheless it has been recognised that in some individual patients, possibly some specific disease subtypes show more dividing cells and chromosomes of greater morphological distinction. This phenomenon is reflected in our finding of more active proliferation in T-cell ALL and more importantly in those cases associated with relapse. They may, although not recognisable by any qualitative risk factor initially, represent a different biological category so far only manifesting higher proliferation and greater risk of relapse or resistance to treatment. One of the traditional risk factors of childhood ALL, the white cell count at diagnosis, may just reflect this phenomenon. However when plotting peripheral against bone marrow cells of ALL at diagnosis there was a very small trend of correlation if any (data not shown).

This is not surprising considering the great variability of peripheral cell-counts in ALL before chemotherapy (viz. the characteristic patients with normal or even lower white cells and a packed bone marrow, frequently accompanied by bone or joint pains).

Obviously the association of higher proliferation at diagnosis with relapse is the most important study result in this group. Ball et al reported similar results from studies using very involved methodology (8). This feature may serve as an additional risk factor for treatment stratification.

$C M L$. This entity may be considered to represent an extreme counterpart of ALL as it is usually diagnosed after long-term growth of the neoplastic cell population with a correspondingly high number of proliferating cells (2) and a comparative ease of cytogenetic analysis. This explains the pioneering role of this disease in tumour cytogenetics. Expectedly our proliferation results in CML are the mirror image of ALL. There was surprising homogeneity between cases of different stages. Even the blast crisis could not be differentiated with this method emphasizing its low sensitivity in this respect. However the biological difference between Philadelphia-positive and -negative CML was apparent in the proliferation results as well.

Aplastic anemia (AA). Acquired aplastic anaemia in childhood is a rare, non-neoplastic life-threatening disorder. No single etiological factor has been identified, instead both toxic and infectious agents are involved in some cases. Accumulating evidence has put the T-cell-related immunopathology of the stem-cell disturbance in focus $(10,11)$.

Chromosome analysis of AA bone marrow has been performed to identify clonal cell populations. Such populations may be an indication of neoplastic disease mimicking AA, or as reported before and after introduction of immunosuppressive therapy in AA, of a preclinical neoplastic clone caused or enhanced by therapy (12-15). Furthermore several numerical and/or structural aberrations have been, albeit infrequently, associated with AA. On the other hand the great majority of patients do not exhibit clonal changes and a large proportion indeed fail to yield any cytogenetic result for the low mitotic activity characteristic of the disorder. This striking feature suggests that the degree of 'visible' proliferation represents an intrinsic parameter of the individual diseased tissue. Therefore, we prospectively analysed the mitotic rates of cytogenetic AA bone marrow samples at diagnosis and during follow-up in order to 
correlate them with subtype, treatment response and outcome. Again there is a great deal of published information available on proliferation properties in the bone marrow failure syndromes based on sophisticated laboratory techniques (16-19). Our aim was however, as for ALL and CML, to extract proliferation data from the yield of routine BM cytogenetic slides.

The proliferation profile of the cohort studied during immunosuppressive therapy evolved towards near normality in the patients responding to treatment (rising cell counts, independence of substitutions with red cells and platelets) (Fig. 10). This illustrates the reliability of the method used for proliferation assessment.

The most relevant finding is the initial difference of the proliferation profiles (and mean scores) between patients with good treatment response and those with poor response (Fig. 12). Although this difference is not sufficiently pronounced to allow allocation of specific risk groups individually, it may provide additional prognostic information, and equally important, shed light on the pathogenetic heterogeneity among AA cases. Gomez-Morales et al (19) demonstrated a correlation between long-term in vitro growth and treatment response. However, we obtained a snapshot picture of BM proliferation measuring after only 24-h incubation (around one cell cycle).

Our observations suggest the following classification of AA patients: i) Group A with initially better BM proliferation and subsequently good IST response: The initial cytopenia may be caused by a mechanism of increased cell loss during differentiation (T-cell autoimmunity?). ii) Group B was characterised by low mitotic activity and worse treatment response: Here a stem cell or precursor defect may appropriately be regarded as the cause of cytopenia and worse IST outcome.

The proliferative deficit associated with low response rates to IST indicates a more basic disorder of cell cycle regulation including increased rates of apoptosis, a greatly reduced stem cell pool etc. Apoptosis seems to be involved in the pathogenesis of AA in different ways: it may contribute to or even cause the marrow suppression observed $(17,20,21)$ and/or special resistance to apoptosis may permit a clone to expand and establish neoplastic disease so typical of aplastic anaemia $(22,23)$.

Subgroups of patients with specific HLA haplotypes have been described which may also indicate pathogenetic heterogeneity $(24,25)$.

In conclusion cytogenetic assessment of bone marrow proliferation in AA at diagnosis provides a parameter which is independent of peripheral cell counts and carries information as to the expected IST response. Moreover, its results illustrate the heterogeneity of AA cases regarding stem cell damage or precursor cell loss respectively.

The simple proliferation assessment presented here is adequate as an initial estimate of disease activity and, with limitations, of disease type to complete biological information obtained at diagnosis.

\section{Acknowledgements}

The authors are indebted to the Wilhelm-Sander-Stiftung (Neustadt/Donau; Grant No. 97.080.1) and the Dr. Sepp-undHanne-Sturm-Stiftung (Munich) for financial support.

\section{References}

1. Ito M, Tsurusawa M, Zha Z, Kawai S, Takasaki Y and Fujimoto T: Cell proliferation in childhood leukemia. Comparison of Ki-67 and proliferating cell nuclear antigen immunocytochemical and DNA flow cytometric analysis. Cancer 69: 2176-2182, 1992.

2. Thiele J, Zirbes TK, Lorenzen J, Kvasnicka HM, Dresbach S, Manich B, Leder LD, Niederle N, Diehl V and Fischer R: Apoptosis and proliferation (PCNA labelling) in CML - a comparative immunohistological study on bone marrow biopsies following interferon and busulfan therapy. J Pathol 181: 316-322, 1997

3. Lin CW, Manshouri T, Jilani I, Neuberg D, Patel K, Kantarjian H, Andreeff M, Estrov Z, Beran M, Keating M, Estey E and Albitar M: Proliferation and apoptosis in acute and chronic leukemias and myelodysplastic syndrome. Leuk Res 26: 551-559, 2002.

4. Kaaijk P, Kaspers GJ, Van Wering ER, Broekema GJ, Loonen AH, Hahlen K, Schmiegelow K, Janka-Schaub GE, Henze G, Creutzig U and Veerman AJ: Cell proliferation is related to in vitro drug resistance in childhood acute leukaemia. Br J Cancer 88: 775-781, 2003.

5. Fuehrer M, Gerusel-Bleck M, Konstantopoulos N, BenderGoetze $\mathrm{C}$ and Walther J-U: FISH analysis of native smears from bone marrow and blood for the monitoring of chimerism and clonal markers after stem cell transplantation in children. Int $\mathbf{J}$ Mol Med 15: 291-297, 2005.

6. Rowley JD: The critical role of chromosome translocations in human leukemias. Annu Rev Genet 32: 495-519, 1998.

7. Cario G, Stanulla M, Fine BM, Teuffel O, Neuhoff NV, Schrauder A, Flohr T, Schafer BW, Bartram CR, Welte K, Schlegelberger B and Schrappe M: Distinct gene expression profiles determine molecular treatment response in childhood acute lymphoblastic leukemia. Blood 105: 821-826, 2005.

8. Ball LM, Pyesmany AF, Yhap M, Lannon CL, Henry M, Laybolt K, Riddell DC and van Velzen D: Related articles. Apoptosis corrected proliferation fraction in childhood ALL is related to karyotype. Adv Exp Med Biol 457: 297-303, 1999.

9. Perez-Vera P, Frias S, Carnevale A, Betancourt M, Mujica M, Rivera-Luna R and Ortiz R: A strategy to detect chromosomal abnormalities in children with acute lymphoblastic leukaemia. J Pediatr Hematol Oncol 26: 294-300, 2004.

10. Young NS: Acquired aplastic anemia. Ann Intern Med 136: 534-546, 2002.

11. Risitano AM, Maciejewski JP, Green S, Plasilova M, Zeng W and Young NS: In vivo dominant immune responses in aplastic anaemia: molecular tracking of putatively pathogenetic T-cell clones by TCR beta-CDR3 sequencing. Lancet 364: 355-364, 2004

12. Mikhailova N, Sessarego M, Fugazza G, Caimo A, De Filippi S, van Lint MT, Bregante S, Valeriani A, Mordini N, Lamparelli T, Gualandi F, Occhini D and Bacigalupo A: Cytogenetic abnormalities in patients with severe aplastic anemia. Haematologica 81: 418-422, 1996.

13. Walther J-U, Chaudhuri Y, Harbott J and Führer M: Monosomy 7 in severe aplastic anaemia (SAA) demonstrated by interphase FISH: its diagnostic and prognostic relevance. J Med Genet 36: S28, 1999.

14. Keung Y, Pettenati M, Cruz J, Powell B, Woodruff R and Buss D: Bone marrow cytogenetic abnormalities of aplastic anemia. Am J Hematol 66: 167-171, 2001.

15. Maciejewski JP, Risitano A, Sloand EM, Nunez O and Young NS: Distinct clinical outcomes for cytogenetic abnormalities evolving from aplastic anemia. Blood 99: 3129-3135, 2002.

16. Haase D and Fonatsch C: Karyotypes and in vitro response to GM-CSF. Analysis of bone marrow cultures in leukemia, myelodysplasia and aplastic anemia. Blut 60: 192-197, 1990.

17. Philpott NJ, Scopes J, Marsh J, Gordon-Smith E and Gibson F: Increased apoptosis in aplastic anemia bone marrow progenitor cells: possible pathophysiologic significance. Exp Hematol 23: 1642-1648, 1995. 
18. Maciejewski J, Sloand E, Sato T, Anderson S and Young N: Impaired hematopoiesis in paroxysmal nocturnal hemoglobinuria/aplastic anemia is not associated with a selective proliferative defect in the glycosylphosphatidylinositolanchored protein-deficient clone. Blood 89: 1173-1181, 1997.

19. Gomez-Morales E, Martinez-Jaramillo G, Sanchez-Valle E, Valencia-Plata I, Arana-Trejo M, Castro M, Pizzuto-Chavez J and Mayani H: Deficient proliferation of myeloid, erythroid, and multipotent progenitor cells in long-term marrow cultures from patients with aplastic anemia treated with immunosuppressive therapy. Am J Hematol 59: 149-155, 1998.

20. Maciejewski J, Selleri C, Sato T, Anderson S and Young N: Increased expression of Fas antigen on bone maarrow CD34 ${ }^{+}$ cells of patients with aplastic anaemia. Br J Haematol 91: 254-52, 1995.

21. Callera F, Garcia A and Falcao R: Fas-mediated apoptosis with normal expression of bcl-2 and p53 in lymphocytes from aplastic anemia. Br J Haematol 100: 698-703, 1998.
22. Horikawa K, Nakakuma H, Kawaguchi T, Iwamoto N, Nagakura S, Kagimoto T and Tagatsuki K: Apoptosis resistance of blood cells from patients with paroxysmal nocturnal hemoglobinuria, aplsatic anemia, and myelodysplastic syndrome. Blood 90: 2716-2722, 1997.

23. De Planque M, Kluin-Neleman H, van Krieken H, Kluin P, Brand A, Beverstock G, Willemze R and van Rood J: Evolution of acquired severe aplastic anaemia to myelodysplasia and subsequent leukaemia in adults. Br J Haematol 70: 55-62, 1988.

24. Nakao S, Takamatsu H, Chuhjo T, Ueda M, Shiobara S, Matsuda T, Kaneshige T and Mizoguchi H: Identification of a specific HLA class II haplotype strongly associated with susceptibility to cyclosporine-dependent aplastic anemia. Blood 84: 4257-4261, 1994.

25. Nimer SD, Ireland P, Meshkinpour A and Frane M: An increased HLA DR2 frequency is seen in aplastic anemia patients. Blood 84: 923-927, 1994. 